\title{
Chiral Light-matter Interaction in Dielectric Photonic Topological Insulators
}

\author{
Sang Soon Oh ${ }^{1}$, Ben Lang ${ }^{2}$, Daryl M. Beggs ${ }^{1}$, Diana L. Huffaker ${ }^{1}$, Matthias Saba ${ }^{3}$, Ortwin Hess ${ }^{3}$ \\ ${ }^{I}$ School of Physics and Astronomy, Cardiff University, Cardiff CF24 3AA, United Kingdom \\ ${ }^{2}$ Department of Electrical and Electronic Engineering, University of Bristol, Bristol BS8 1UB, United Kingdom \\ ${ }^{3}$ Department of Physics, Imperial College London, London SW7 2AZ, United Kingdom \\ OhS2@cardiff.ac.uk
}

\begin{abstract}
A unidirectional chiral edge mode in photonic topological insulators can be selectively excited by a circularly-polarized dipole source. We show that the directionality is also determined by the position of the dipole source.

OCIS codes: (270.1670) Coherent optical effects; (130.5296) Photonic crystal waveguides
\end{abstract}

\section{Introduction}

A photonic topological insulator (PTI) is a new type of photonic structure that can control the light with more freedom than conventional optical devices or photonic crystals [1]. One of the interesting features of photonic topological insulator is the unidirectional chiral edge mode, which appears at the interface between two different PTIs. There are two types of PTIs: time-reversal symmetry broken and time-reversal symmetric PTIs. Time-reversal symmetry broken PTIs include magnetic materials, and they can display a non-reciprocal wave propagation allowing only one unidirectional waveguide mode. In contrast, the time-reversal symmetric PTIs cannot be non-reciprocal but they allow two spin-locked counter-propagating modes mimicking the quantum spin Hall effect.

Recently, Wu and Hu proposed a PTI composed of a hexagonal array of honeycombs of dielectric rods [2]. In the PTI, there exists two counter-propagating modes which can be associated with two pseudo-spin states. These chiral edges modes were shown to be robust to defects such as dislocations of the lattice and they showed how the two circularly polarized light sources can excite the two counter-propagating modes selectively. It has been shown that there is close link between the polarization of the dipole source and the modes, but it is not still clear how the position of the source influences the coupling between the source and the modes.

In this work, we investigate the coupling between the dipole source and the chiral modes by calculating the local chirality of the propagating modes [3]. The local chirality $(u / v)$, that is defined as the ratio major and minor axes $(u, v)$ of the polarization ellipse, is calculated from the magnetic fields of the chiral modes. Based on the calculated local chirality, we investigate how the source position is associated with the selective excitation of chiral edge modes. This position effect would be very important in understanding the light-matter interaction for a quantum dot embedded in PTIs.

\section{Chirality of edge modes}

We consider a hexagonal array of honeycomb array of dielectric rods (see Fig. 1(a)). The lattice constant of the hexagonal array $a$ is fixed to 1 , the size of honeycomb in a unit cell is described by $\mathrm{R} 1, \mathrm{R} 2$. The dielectric constant of the dielectric rod is 11.7. Note that TE modes are considered here but the concept of chirality of the edge modes can

(a)

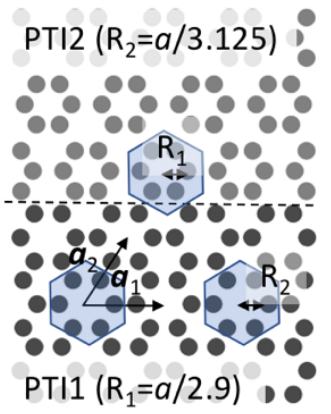

(b)

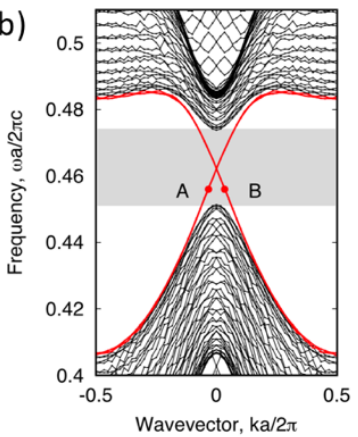

(c)

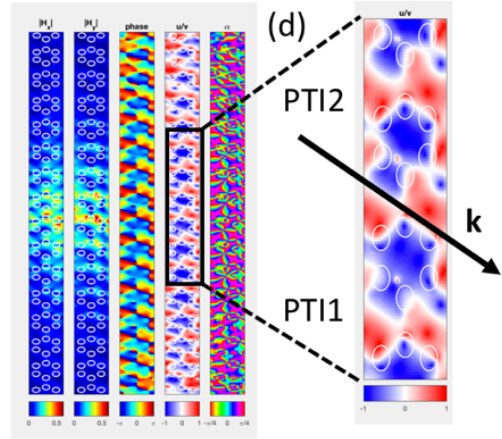

Figure 1. (a) A hexagonal array of honeycomb with different sizes $R_{1}$ and $R_{2} . a_{1}$ and $\boldsymbol{a}_{2}$ are the lattice vectors of the hexagonal array and $a$ is the lattice constant. (b) Photonic band diagram showing two counter-propagating edge modes (c) Magnetic field components profiles, phases, chirality $(u / v)$ and orientation angle $(\alpha)$ for the edge mode at $k=0.04 c / a$ denoted by B in (b). $\mathbf{k}$ is the wavevector of the edge mode. 
be similarly applied to TM modes. Using the photonic mode solver $m p b$ [4], we calculated the band structure and found that the frequencies of the edge modes are between 0.450-0.472 cla (Fig. 1(b)). As shown in Fig. 1(c), the magnetic fields are localized at the interface of two PTIs. From the magnetic field components $\left(H_{x}, H_{y}\right)$ of the edge modes, we can calculate the chirality of the mode for $\mathrm{H}$ field can be calculated using the Stokes parameters.

Figure 1(c) shows the calculated magnetic field components, the phase difference between the longitudinal and transverse components of magnetic fields and the chirality $(u / v)$. It is interesting to see that chirality in the area between the two different PTIs has almost the same sign of values (close to +1 , shown in red) in contrast to the photonic crystal case [3]. However, at about a half period away from the interface, the chirality has an opposite sign (shown in blue). This implies that we need different circular polarizations at the two positions (e.g. points denoted by 0 and 1 in Fig. 2) to excite edge modes propagating in the same direction. Alternatively, the same circularly polarized dipoles at the two positions would excite edge modes propagating in the opposite direction.

\section{One-way mode excitation by a polarized dipole source}

To confirm the spin-dependent directionality, we performed the FDTD simulations using the open source software meep [5]. In the FDTD simulations, we used the same parameters with the $m p b$ simulations and we located an magnetic dipole source which has magnetic fields in the $x y$ plane $H_{x} \hat{x}+H_{y} e^{i \phi} \hat{y}$ with $H_{x}=H_{y}, \phi=\pi / 2$ for a positive spin and $H_{x}=H_{y}, \phi=-\pi / 2$ for a negative spin. As shown in Fig. 2, the sources with the same polarization (positive spin) at position 1 and position 2 excite one-way edge modes propagating to the left and the right, respectively. Since the absolute values of chirality are close to the unity, the mode excitation at these two points are similar to the ones at the points near C-point in Ref. 3. In fact, the two counter-propagating modes are excited simultaneously but with different amplitudes.

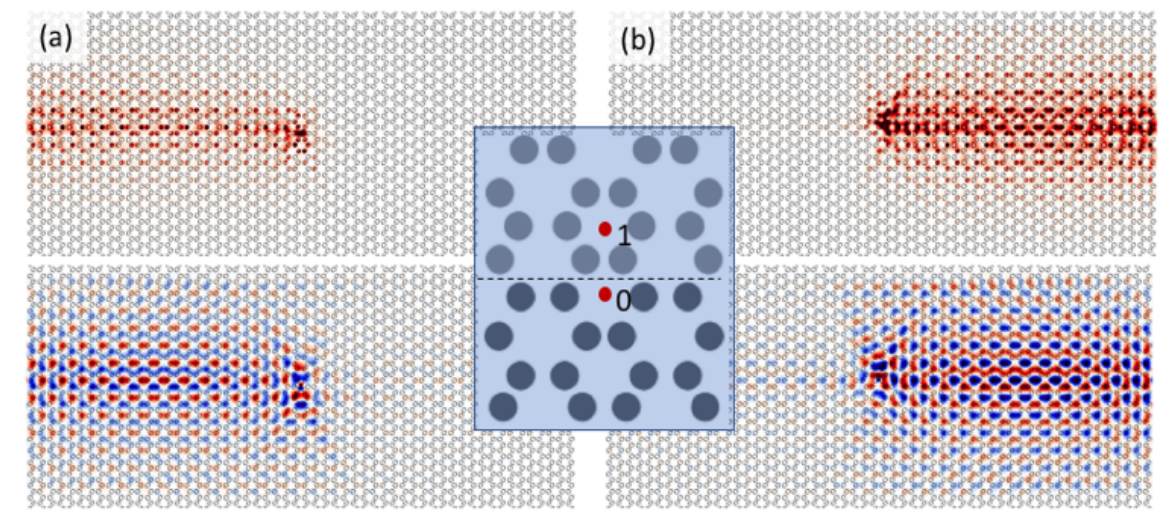

Figure 2. Snapshots of power (top) and electric field ( $E_{z}$, bottom) of edge modes excited by a polarized magnetic dipole source (positive spin) (a) at position 0 and (b) at position 1.

\section{Conclusions}

Using the chirality map for a topological edge mode in a dielectric photonic topological insulator, we were able to show the source-position dependent excitation of one-way edge modes. We numerically demonstrated the unidirectional edge modes with polarized magnetic dipole sources.

[1] L. Lu, J. D. Joannopoulos, and M. Soljačić, “Topological photonics,” Nat. Photonics 8, 821 (2014).

[2] L.-H. Wu and X. Hu, "Scheme for Achieving a Topological Photonic Crystal by Using Dielectric Material," Phys. Rev. Lett. 114, 223901 (2015).

[3] A. B. Young, A. C. T. Thijssen, D. M. Beggs, P. Androvitsaneas, L. Kuipers, J. G. Rarity, S. Hughes, and R. Oulton, "Polarization Engineering in Photonic Crystal Waveguides for Spin-Photon Entanglers," Phys. Rev. Lett. 115, 153901 (2015).

[4] S. G. Johnson and J. D. Joannopoulos, "Block-iterative frequency-domain methods for Maxwell's equations in a planewave basis," Optics Express 8, 173-190 (2001).

[5] A.F. Oskooi, D. Roundy, M. Ibanescu, P. Bermel, J.D. Joannopoulos, and S.G. Johnson, "MEEP: A flexible free-software package for electromagnetic simulations by the FDTD method, Computer Physics Communications," 181, 687-702 (2010). 